\title{
Stability aspects of arithmetic functions, II
}

\author{
by
}

\section{Tomasz KochaneK (Katowice)}

Introduction. The present paper is a continuation of [7]. We deal with the stability problem for arithmetic additive and multiplicative functions in the sense of Hyers and Ulam (see [11]). Let $f: \mathbb{N} \rightarrow \mathbb{C}$. The two basic hypotheses we are interested in are:

$$
\begin{gathered}
x, y \in \mathbb{N},(x, y)=1 \Rightarrow|f(x y)-f(x)-f(y)| \leq \varepsilon, \\
x, y \in \mathbb{N},(x, y)=1 \Rightarrow\left|\frac{f(x y)}{f(x) f(y)}-1\right| \leq \varepsilon
\end{gathered}
$$

with a fixed $\varepsilon \geq 0$. Here, and in what follows, the symbol $(x, y)$ stands for the greatest common divisor of $x$ and $y$.

In the case of assumption (A) we expect the existence of an arithmetic additive function $\tilde{f}$ such that the difference $f-\tilde{f}$ is globally bounded by $\delta(\varepsilon)$ with $\lim _{\varepsilon \rightarrow 0} \delta(\varepsilon)=0$. A couple of results in that spirit was obtained by the author in [7] under some additional assumptions.

The proof of one of them uses a technique, based on the notion of Banach limit, reducing the conditional functional inequality (A) to an unconditional one. This allows one to apply the classical Hyers theorem [4]. In the present paper we propose a simple proof of a somewhat stronger version of that result (see Theorem 1.10 below). On the other hand, we apply the original method of proof to establish Theorem 1.11 which asserts, roughly speaking, that if $f$ satisfies (A), then it can be well approximated by a concrete arithmetic additive function on a "large" subset of $\mathbb{N}$.

Another approach to our problem is based on the classical Farkas lemma which yields a sufficient condition for solvability of systems of linear inequalities. We give two theorems whose proofs rely on this idea. In the first one (Theorem 1.7) we replace assumption (A) by a more general but still quite

2000 Mathematics Subject Classification: Primary 11K65, 39B82.

Key words and phrases: arithmetic functions, almost additive functions, Hyers-Ulam stability, conditional functional equation. 
natural condition. Namely, we suppose that every difference of the form

$$
\sum_{i=1}^{m} f\left(x_{i}\right)-\sum_{j=1}^{n} f\left(y_{j}\right)
$$

is estimated (in absolute value) by $(m+n) \varepsilon$, provided it "annihilates" every prime power in the following sense: for each prime $p$ and $k \in \mathbb{N}$ we have

$$
\sum_{p^{k} \| x_{i}} 1=\sum_{p^{k} \| y_{j}} 1
$$

$\left(p^{k} \| x \Leftrightarrow p^{k} \mid x\right.$ but $\left.p^{k+1} \nmid x\right)$. Observe that, indeed, it is a kind of generalization of hypothesis (A), since every difference $f(x y)-f(x)-f(y)$ with $x$ and $y$ coprime "annihilates" every prime power in the above sense.

Theorem 1.8 below, also based on the Farkas lemma, concerns the situation where we replace the constant $\varepsilon$ in (A) by some "control" function $\varphi(x, y)$. As a result, we obtain an approximation

$$
|f(x)-\widetilde{f}(x)| \leq \psi(x), \quad x \in \mathbb{N},
$$

with an arithmetic additive function $\widetilde{f}$, where $\varphi$ and $\psi$ are suitably joined.

All the results mentioned above require either some kind of modification of condition (A), or some extra assumption on the function $f$. But is there an arithmetic additive function lying near $f$ to within $\delta(\varepsilon)$ if $f$ is supposed to satisfy only condition (A)? It appears that this question is not far away from an analogous question stated for finitely additive set functions, where this problem is completely solved by the brilliant theorem of N. J. Kalton and J. W. Roberts [5, Theorem 4.1]. Our first proposition is a slight modification of their result and has an immediate translation into an affirmative answer for the question of stability for arithmetic additive functions with $\delta(\varepsilon) \equiv K \varepsilon$, where $K \leq 89 / 2$ is the Kalton-Roberts constant (Theorem 1.5).

The problem of determining the optimal constant is open and seems to be difficult. The reason is that the value of $K$ follows from quite subtle considerations of combinatorial nature (see [9]).

As already mentioned, all previously announced results concerning the additive case require additional or modified assumptions (in contrast to Theorem 1.5). Despite this fact, they are not weaker than Theorem 1.5 (at least formally). Firstly, in all of them the error constant is smaller than $K \varepsilon$ (with the exception of Theorem 1.8 where the error is estimated by a function). Secondly, in some cases we know exactly the additive function $\widetilde{f}$ which approximates $f$ (usually $f$ and $\widetilde{f}$ coincide on the set of all prime powers). These are some profits from adopting additional conditions upon $f$.

In the last section we translate our assertions concerning the additive case into the multiplicative case. Some results on the stability problem for arithmetic multiplicative functions were obtained earlier in [8] and [7]. An 
assumption similar to (M) was investigated by R. Ger and P. Šemrl [2] in the case of ordinary multiplicative mappings; $\mathrm{R}$. Ger [1] was the first to indicate that such a condition is the most natural one.

1. Stability results in the additive case. Let $\mathbb{P}$ denote the set of all primes, and set $\mathbb{S}=\left\{p^{k}: p \in \mathbb{P}, k \in \mathbb{N}\right\}$ and $\omega(x)=\#\{p \in \mathbb{P}: p \mid x\}$ for $x \in \mathbb{N}$. For $n \in \mathbb{N}$ we define $\omega_{\prec n}=\{x \in \mathbb{N}: \omega(x) \prec n\}$ where $\prec$ may be any binary relation on the integers.

We introduce a notion of "largeness" in $\mathbb{N}$, which will turn out to be quite natural for our assertions.

Definition 1.1. We say that a set $A \subset \mathbb{N}$ is large if for every $n \in \mathbb{N}$ there is a finite set $P_{n} \subset \mathbb{P}$ such that

$$
\left(\mathbb{N} \backslash\left(P_{n} \cdot \mathbb{N}\right)\right) \cap \omega_{=n} \subset A .
$$

Lemma 1.2. Assume that a set $A \subset \mathbb{N}$ has the property that $A \cap B \neq \emptyset$ for each infinite set $B \subset \mathbb{N}$ satisfying the following two conditions:

(i) if $x, y \in B, x \neq y$, then $(x, y)=1$;

(ii) if $x, y \in B$, then $\omega(x)=\omega(y)$.

Then (and only then) $A$ is large. Moreover, if we omit condition (ii) in the above hypothesis, then (and only then) there exists a finite set $P \subset \mathbb{P}$ such that

$$
\mathbb{N} \backslash(P \cdot \mathbb{N}) \subset A \cup\{1\} .
$$

In that case the set $A$ has a positive natural density.

Proof. The "only then" parts of our statement are obvious.

We are going to prove both "then" assertions simultaneously, but first observe that condition (ii) may be easily replaced by the formally weaker one: $\sup _{x \in B} \omega(x)<\infty$.

Fix $\kappa \in \mathbb{N} \cup\{\infty\}$; the case of $\kappa$ finite will correspond to the first assertion, and $\kappa=\infty$ to the second.

Choose any $x_{1} \notin A \cup\{1\}, x \in \omega_{<\kappa}$ (if no such $x_{1}$ exists, then we are done). Let $Q_{1}$ be the set of all prime factors of $x_{1}$. Assuming that we have already chosen mutually coprime numbers $x_{1}, \ldots, x_{n} \notin A \cup\{1\}$, all belonging to $\omega_{<\kappa}$, we pick

$$
x_{n+1} \notin A \cup\{1\} \cup\left(\bigcup_{i=1}^{n} Q_{i}\right) \cdot \mathbb{N}
$$

$\left(Q_{i}\right.$ being the set of all prime factors of $\left.x_{i}\right)$ such that $x_{n+1} \in \omega_{<\kappa}$, provided any such $x_{n+1}$ exists. In view of our assumption, the process must come to a halt after a finite number of steps. This means that for a certain $N \in \mathbb{N}$ 
we have

$$
\omega_{<\kappa} \subset A \cup\{1\} \cup\left(\bigcup_{i=1}^{N} Q_{i}\right) \cdot \mathbb{N},
$$

i.e.,

$$
\left(\mathbb{N} \backslash\left(P_{\kappa} \cdot \mathbb{N}\right)\right) \cap \omega_{<\kappa} \subset A \cup\{1\},
$$

where $P_{\kappa}:=\bigcup_{i=1}^{N} Q_{i}$. This proves that $A$ is large in the case $\kappa<\infty$, whereas in the opposite case we have (1.2) with $P:=P_{\infty}$.

Now we proceed to the proof of our last assertion. Let $\bigcup_{n \in \mathbb{N}} P_{n}=$ $\left\{p_{1}, \ldots, p_{n}\right\}$. We have

$$
\mathbb{N} \backslash\left(p_{1} \mathbb{N} \cup \cdots \cup p_{n} \mathbb{N}\right) \subset A \cup\{1\} .
$$

It is clear that for the natural density $d$ we have

$$
\begin{aligned}
d\left(p_{1} \mathbb{N} \cup \cdots \cup p_{n} \mathbb{N}\right) & =\sum_{1 \leq i \leq n} \frac{1}{p_{i}}-\sum_{1 \leq i<j \leq n} \frac{1}{p_{i} p_{j}}+\cdots \pm \frac{1}{p_{1} \cdot \cdots \cdot p_{n}} \\
& =1-\prod_{i=1}^{n}\left(1-\frac{1}{p_{i}}\right) .
\end{aligned}
$$

Hence $d(A) \geq \prod_{i=1}^{n}\left(1-1 / p_{i}\right)>0$.

EXAMPLE 1.3. We show that the last assertion of Lemma 1.2 fails to hold if we restrict the supposition $A \cap B \neq \emptyset$ to only those infinite sets $B \subset \mathbb{N}$ for which both (i) and (ii) are valid.

To this end, let $p_{1}, p_{2}, \ldots$ be any enumeration of all prime numbers. Now, define

$$
A_{n}=\left(\mathbb{N} \backslash\left(p_{1} \mathbb{N} \cup \cdots \cup p_{n} \mathbb{N}\right)\right) \cap \omega_{\leq n} \quad \text { for } n \in \mathbb{N}
$$

and $A=\bigcup_{n=1}^{\infty} A_{n}$. We shall prove that $d(\mathbb{N} \backslash A)=1$.

Observe that

$$
\mathbb{N} \backslash A=\left\{x \in \mathbb{N}: x \in p_{1} \mathbb{N} \cup \cdots \cup p_{\omega(x)} \mathbb{N}\right\} .
$$

Hence, for a fixed $n \in \mathbb{N}$, we have

$$
\left(p_{1} \mathbb{N} \cup \cdots \cup p_{n} \mathbb{N}\right) \cap \omega_{\geq n} \subset \mathbb{N} \backslash A .
$$

The set on the left-hand side has the same density as $p_{1} \mathbb{N} \cup \cdots \cup p_{n} \mathbb{N}$, since $d\left(\omega_{\geq n}\right)=1$ (because $d\left(\omega_{=n}\right)=0$ for all $n \in \mathbb{N}$, which follows, e.g., from the Hardy-Ramanujan theorem [3]). However,

$$
d\left(p_{1} \mathbb{N} \cup \cdots \cup p_{n} \mathbb{N}\right)=1-\prod_{i=1}^{n}\left(1-\frac{1}{p_{i}}\right) \stackrel{n \rightarrow \infty}{\longrightarrow} 1,
$$

which implies what we have claimed.

In what follows $\varepsilon$ always stands for an arbitrary non-negative number, unless otherwise stated. 
The proof of the following modification of the Kalton-Roberts theorem [5, Theorem 4.1] is only sketched, since it is almost the same as the original one.

Proposition 1.4. Let $\Omega$ be a finite set and let $\mathcal{R}=\left\{\Omega_{1}, \ldots, \Omega_{N}\right\}$ be a collection of non-empty mutually disjoint sets with union $\Omega$. Define $\mathcal{A} \subset 2^{\Omega}$ as

$$
\mathcal{A}=\left\{A \subset \Omega: \forall_{1 \leq i \leq N} \#\left(A \cap \Omega_{i}\right) \leq 1\right\} .
$$

Assume that a function $\nu: \mathcal{A} \rightarrow \mathbb{R}$ satisfies

$$
(A, B, A \cup B \in \mathcal{A} \text { and } A \cap B=\emptyset) \Rightarrow|\nu(A \cup B)-\nu(A)-\nu(B)| \leq \varepsilon .
$$

Then there exists a function $\mu: \mathcal{A} \rightarrow \mathbb{R}$ such that

$$
(A, B, A \cup B \in \mathcal{A} \text { and } A \cap B=\emptyset) \Rightarrow \mu(A \cup B)=\mu(A)+\mu(B)
$$

and

$$
|\nu(A)-\mu(A)| \leq K \varepsilon, \quad A \in \mathcal{A} .
$$

Proof. Every function $\mu: \mathcal{A} \rightarrow \mathbb{R}$ with property (1.5) will be called $\mathcal{R}$ additive. For any $f: \mathcal{A} \rightarrow \mathbb{R}$ define

$$
V(f)=\max _{A, B \in \mathcal{A}}(f(A)-f(B)) .
$$

If $\sim$ is the equivalence relation in $\mathbb{R}^{\mathcal{A}}$ given by $(f \sim g) \Leftrightarrow(f-g$ is constant $)$, then $\left(\mathbb{R}^{\mathcal{A}} / \sim, \rho\right)$ is a metric space with $\rho\left([f]_{\sim},[g]_{\sim}\right):=V(f-g)$. Let $\mathcal{M}$ be the set of all $[\mu]_{\sim}$ where $\mu$ is $\mathcal{R}$-additive. Take a sequence of $\left[\mu_{n}\right]_{\sim} \in \mathcal{M}$, $n \in \mathbb{N}$, such that

$$
\operatorname{dist}\left([\nu]_{\sim}, \mathcal{M}\right)=: d \leq \rho\left([\nu]_{\sim},\left[\mu_{n}\right]_{\sim}\right) \leq d+1 / n .
$$

The set $\left\{\mu_{n}\right\}_{n \in \mathbb{N}}$ is bounded at each point of $\mathcal{A}$, thus it is contained in a compact subset of $\mathbb{R}^{\mathcal{A}}$. Let $\mu$ be the limit of some convergent subsequence of $\left\{\mu_{n}\right\}_{n \in \mathbb{N}}$. Then $\mu$ is $\mathcal{R}$-additive and $V(\nu-\mu)=d$.

Let $g=\nu-\mu$ and

$$
a=\max _{A \in \mathcal{A}} g(A), \quad b=-\min _{A \in \mathcal{A}} g(A) ;
$$

we may suppose that $a \geq b$. We shall show that $a \leq K \varepsilon$. Take a set $S \in \mathcal{A}$ such that $g(S)=a$. The rest of the argument is exactly as in the proof of Kalton and Roberts. We define the same submeasure $\varphi: 2^{S} \rightarrow[0, \infty)$ (since $S \in \mathcal{A}$ implies $2^{S} \subset \mathcal{A}$, everything works). Then, with the aid of Kelley's theorem [6, Corollary 6] and [5, Lemma 3.1], we obtain the desired estimate.

THEOREM 1.5. If $f: \mathbb{N} \rightarrow \mathbb{R}$ satisfies (A), there is a real arithmetic additive function $\tilde{f}$ such that $|f(x)-\widetilde{f}(x)| \leq K \varepsilon$ for $x \in \mathbb{N}$. 
Proof. Let

$$
\mathcal{Z}=\prod_{s \in \mathbb{S}}[f(s)-K \varepsilon, f(s)+K \varepsilon]
$$

then, by Tikhonov's theorem, $\mathcal{Z}$ is a compact space. If $x \in \mathbb{N}$ with $x \geq 2$ has a canonical factorization $x=s_{1} \cdot \ldots \cdot s_{m}$ (with $s_{i} \in \mathbb{S}$ mutually coprime), then we define

$$
\mathcal{Z}_{x}=\left\{(\xi(s))_{s \in \mathbb{S}} \in \mathcal{Z}:\left|f(x)-\left(\xi\left(s_{1}\right)+\cdots+\xi\left(s_{m}\right)\right)\right| \leq K \varepsilon\right\} .
$$

Plainly, $\mathcal{Z}_{x}$ is a closed subspace of $\mathcal{Z}$.

Fix a finite number of natural numbers $x_{1}, \ldots, x_{n} \geq 2$ with canonical factorizations

$$
x_{i}=s_{i 1} \cdot \ldots \cdot s_{i m_{i}}, \quad i=1, \ldots, n,
$$

and define

$$
\Omega=\left\{s_{i j}: i=1, \ldots, n, j=1, \ldots, m_{i}\right\} .
$$

Let $\mathcal{R}=\left\{\Omega_{1}, \ldots, \Omega_{N}\right\}$ denote the partition of $\Omega$ for which the following is true: $s_{i j}, s_{k l}$ belong to the same part of $\mathcal{R}$ iff $\left(s_{i j}, s_{k l}\right)>1$. Let $\mathcal{A}$ be defined by (1.3). Now, we may apply Proposition 1.4 to the function $\nu: \mathcal{A} \rightarrow \mathbb{R}$ given by

$$
\nu(A)=f\left(\prod_{a \in A} a\right), \quad A \in \mathcal{A},
$$

since assumption (A) implies condition (1.4). There exists an $\mathcal{R}$-additive function $\mu$ satisfying (1.6). In particular, putting $A=\left\{s_{i 1}, \ldots, s_{i m_{i}}\right\}$ for $i=1, \ldots, n$ we obtain

$$
\left|f\left(x_{i}\right)-\left(\mu\left(\left\{s_{i 1}\right\}\right)+\cdots+\mu\left(\left\{s_{i m_{i}}\right\}\right)\right)\right| \leq K \varepsilon,
$$

whereas taking $A=\left\{s_{i j}\right\}$ for $i=1, \ldots, n, j=1, \ldots, m_{i}$ we get

$$
\mu\left(\left\{s_{i j}\right\}\right) \in\left[f\left(s_{i j}\right)-K \varepsilon, f\left(s_{i j}\right)+K \varepsilon\right] .
$$

Hence the sequence $(\xi(s))_{s \in \mathbb{S}}$ defined by

$$
\xi(s)= \begin{cases}\mu(\{s\}) & \text { if } s \in \Omega, \\ f(s) & \text { otherwise }\end{cases}
$$

belongs to $\bigcap_{i=1}^{n} \mathcal{Z}_{x_{i}}$. Consequently, the family $\left\{\mathcal{Z}_{x}: x \geq 2\right\}$ has the finite intersection property, and thus there exists

$$
(\xi(s))_{s \in \mathbb{S}} \in \bigcap_{x \geq 2} \mathcal{Z}_{x} .
$$

It remains to define $\widetilde{f}$ as the unique arithmetic additive function satisfying $\widetilde{f}(s)=\xi(s)$ for $s \in \mathbb{S}$. For $x \geq 2$ the inequality $|f(x)-\widetilde{f}(x)| \leq K \varepsilon$ follows directly from (1.7), while for $x=1$ it is obvious, since $\widetilde{f}(1)=0$ and $|f(1)|$ $\leq \varepsilon$. 
REMARK 1.6. The estimate given in Theorem 1.5 may be improved in the following way. If $f: \mathbb{N} \rightarrow \mathbb{C}$ satisfies (A), then there is an arithmetic additive function $\tilde{f}$ such that for every $\eta>0$ the set

$$
\{x \in \mathbb{N}:|f(x)-\widetilde{f}(x)|<\varepsilon+\eta\}
$$

has positive natural density.

Indeed, an analogue of Theorem 1.5 is valid for complex-valued functions $f$ and $\widetilde{f}$ after replacing $K \varepsilon$ by $\sqrt{2} K \varepsilon$. Considering an arbitrary function $f: \mathbb{N} \rightarrow \mathbb{C}$ instead of the difference $f-\widetilde{f}$ we shall prove that if $f$ is bounded and satisfies (A), then the above assertion is valid for every $\eta>0$ and the set $A_{\eta}:=\{x \in \mathbb{N}:|f(x)|<\varepsilon+\eta\}$. Furthermore, by virtue of Lemma 1.2, it is enough to show that $A_{\eta} \cap B \neq \emptyset$ for each infinite set $B \subset \mathbb{N}$ with pairwise coprime elements.

Suppose on the contrary that $B$ is a set with the properties in question and $|f(x)| \geq \varepsilon+\eta$ for each $x \in B$. Choose arbitrarily $\alpha_{1} \in(1,2)$. The region $\{z \in \mathbb{C}:|z| \geq \varepsilon+\eta\}$ may be partitioned into finitely many sectors in such a way that for all complex numbers $z_{1}, z_{2}$ belonging to the same sector we have $\left|z_{1}+z_{2}\right| \geq 2 \varepsilon+\alpha_{1} \eta$. There is an infinite set $\left\{x_{1}, x_{2}, \ldots\right\} \subset B$ whose image under $f$ lies in one of these sectors. By inequality (A), we infer that $\left|f\left(x_{i} x_{i+1}\right)\right|>\varepsilon+\alpha_{1} \eta$ for $i \in \mathbb{N}$.

Now, repeat this process replacing $B$ by the set $\left\{x_{1} x_{2}, x_{3} x_{4}, \ldots\right\}$ and $\eta$ by $\alpha_{1} \eta$. Taking a sequence $\left(\alpha_{n}\right)_{n \in \mathbb{N}}$ such that $\prod_{n=1}^{\infty} \alpha_{n}=\infty$ we arrive at a contradiction with the assumption that $f$ is bounded.

TheOREM 1.7. Assume that a function $f: \mathbb{N} \rightarrow \mathbb{R}$ satisfies

$$
\left|\sum_{i=1}^{m} f\left(x_{i}\right)-\sum_{j=1}^{n} f\left(y_{j}\right)\right| \leq(m+n) \varepsilon
$$

for every $x_{1}, \ldots, x_{m}, y_{1}, \ldots, y_{n} \in \mathbb{N}$ such that

$$
\sum_{p^{k} \| x_{i}} 1=\sum_{p^{k} \| y_{j}} 1 \quad \text { for all } p \in \mathbb{P}, k \in \mathbb{N} .
$$

Then there is a real arithmetic additive function $\widetilde{f}$ such that $|f(x)-\widetilde{f}(x)| \leq \varepsilon$ for $x \in \mathbb{N}$.

Proof. As is clear from the proof of Theorem 1.5, it is enough to show that for any finite set $\Omega \subset \mathbb{S}$ there exists a vector $\left(x_{a}\right)_{a \in \Omega} \in \mathbb{R}^{\# \Omega}$ which yields a solution to each of the inequalities

$$
\left|f\left(\prod_{a \in A} a\right)-\sum_{a \in A} x_{a}\right| \leq \varepsilon, \quad A \in \mathcal{A},
$$

where $\mathcal{A}$ is defined as above. Equivalently, we shall show that the system of linear inequalities 
is consistent.

$$
\left\{\begin{array}{rl}
\sum_{a \in A} x_{a} & \leq f\left(\prod_{a \in A} a\right)+\varepsilon, \\
-\sum_{a \in A} x_{a} \leq-f\left(\prod_{a \in A} a\right)+\varepsilon,
\end{array} \quad A \in \mathcal{A},\right.
$$

To this end we apply [10, Theorem 22.1]. Suppose $\left(\alpha_{A}\right)_{A \in \mathcal{A}},\left(\beta_{A}\right)_{A \in \mathcal{A}}$ are non-negative numbers satisfying

$$
\sum_{a \in A}\left(\alpha_{A}-\beta_{A}\right)=0, \quad a \in \Omega ;
$$

the question is whether

$$
\sum_{A \in \mathcal{A}}\left(\alpha_{A}-\beta_{A}\right) f\left(\prod_{a \in A} a\right)+\varepsilon \sum_{A \in \mathcal{A}}\left(\alpha_{A}+\beta_{A}\right) \geq 0 .
$$

Putting $\gamma_{A}=\alpha_{A}-\beta_{A}$ for $A \in \mathcal{A}$, we may rewrite equation (1.10) as

$$
\sum_{a \in A} \gamma_{A}=0, \quad a \in \Omega
$$

if we show that

$$
\left|\sum_{A \in \mathcal{A}} \gamma_{A} f\left(\prod_{a \in A} a\right)\right| \leq \varepsilon \sum_{A \in \mathcal{A}}\left|\gamma_{A}\right|,
$$

then (1.11) will follow.

First, observe that it is enough to prove that (1.12) implies (1.13) in the case where every $\gamma_{A}$, for $A \in \mathcal{A}$, is rational. Indeed, since all equations in (1.12) have rational coefficients, the hyperplane $H_{\Omega} \subset \mathbb{R}^{\# \mathcal{A}}$ determined by these equations has the property that $H_{\Omega} \cap \mathbb{Q}^{\# \mathcal{A}}$ is dense in $H_{\Omega}$. Thus, if we show (1.13) for all $\left(\gamma_{A}\right)_{A \in \mathcal{A}} \in H_{\Omega} \cap \mathbb{Q}^{\# \mathcal{A}}$, then, obviously, it would also be valid in $H_{\Omega}$.

Now, fix $\left(\gamma_{A}\right)_{A \in \mathcal{A}} \in H_{\Omega} \cap \mathbb{Q}^{\# \mathcal{A}}$. After multiplying both sides of (1.13) by a common multiple of the denominators of $\left(\gamma_{A}\right)_{A \in \mathcal{A}}$ and repeating the terms $f\left(\prod_{a \in A} a\right)$ as required we arrive at an inequality of form (1.8) for which condition (1.9) is guaranteed by the fact that $\left(\gamma_{A}\right)_{A \in \mathcal{A}}$ satisfies (1.12).

Let $\mathcal{P}$ be the set of all pairs of relatively prime natural numbers.

Theorem 1.8. Given $\varphi: \mathcal{P} \rightarrow[0, \infty)$ assume that a function $f: \mathbb{N} \rightarrow \mathbb{R}$ satisfies

$$
x, y \in \mathbb{N},(x, y)=1 \Rightarrow|f(x y)-f(x)-f(y)| \leq \varphi(x, y) .
$$

Let $\psi: \mathbb{N} \rightarrow[0, \infty)$ be any function satisfying

$$
\psi(x z)+\psi(y z) \geq \psi(x)+\psi(y)+\varphi(x, z)+\varphi(y, z)
$$

for all $x, y, z \in \mathbb{N}$ such that $(x, y),(y, z),(z, x) \in \mathcal{P}$. Then there is a real arithmetic additive function $\widetilde{f}$ such that $|f(x)-\widetilde{f}(x)| \leq \psi(x)$ for $x \in \mathbb{N}$. 
Proof. The idea is similar to the previous one. Now we are interested in the consistency of the system

$$
\left\{\begin{aligned}
\sum_{a \in A} x_{a} \leq f\left(\prod_{a \in A} a\right)+\psi\left(\prod_{a \in A} a\right), & \\
-\sum_{a \in A} x_{a} \leq-f\left(\prod_{a \in A} a\right)+\psi\left(\prod_{a \in A} a\right), & A \in \mathcal{A} .
\end{aligned}\right.
$$

Again applying [10, Theorem 22.1] we shall prove that for every $\left(\gamma_{A}\right)_{A \in \mathcal{A}}$ $\in H_{\Omega}$ (which can be supposed to lie in $\mathbb{Z}^{\# \mathcal{A}}$ ),

$$
\left|\sum_{A \in \mathcal{A}} \gamma_{A} f\left(\prod_{a \in A} a\right)\right| \leq \sum_{A \in \mathcal{A}}\left|\gamma_{A}\right| \psi\left(\prod_{a \in A} a\right) .
$$

This can be rewritten in the form

$$
\left|\sum_{i=1}^{m} \lambda_{i} f\left(x_{i}\right)\right| \leq \sum_{i=1}^{m}\left|\lambda_{i}\right| \psi\left(x_{i}\right),
$$

where $x_{1}, \ldots, x_{m} \in \mathbb{N}$ are pairwise different and $\lambda_{1}, \ldots, \lambda_{m} \in \mathbb{Z}$ satisfy

$$
\sum_{p^{k} \| x_{i}} \lambda_{i}=0 \quad \text { for all } p \in \mathbb{P}, k \in \mathbb{N} .
$$

We prove (1.16) by induction on $c:=\sum_{i=1}^{m}\left|\lambda_{i}\right| \omega\left(x_{i}\right)$. For $c=0$ we have $m=1, x_{1}=1$. Inequality (1.16) then takes the form $|\lambda f(1)| \leq|\lambda| \psi(1)$, which is valid, since (1.14) and (1.15) imply $f(1)=0$ (put $x=y=z=1$ ). Now, assume that $c>0$ and our assertion holds true if $\sum_{i=1}^{m}\left|\lambda_{i}\right| \omega\left(x_{i}\right)<c$. Since $f(1)=0$, we may suppose that all $x_{i}$ 's are different from 1 . Choose any prime power $p^{k} \| x_{1}$. In view of (1.17), there is $j \in\{2, \ldots, m\}$ with $p^{k} \| x_{j}$ and $\lambda_{1} \lambda_{j}<0$. After renumbering we may assume that $j=2$ and $\lambda_{1}>0$, $\lambda_{2}<0$. By virtue of (1.14), we have

$$
\begin{aligned}
\mid f\left(x_{1}\right)-f\left(x_{2}\right)- & f\left(x_{1} /\left(x_{1}, x_{2}\right)\right)+f\left(x_{2} /\left(x_{1}, x_{2}\right)\right) \mid \\
\leq & \left|f\left(x_{1}\right)-f\left(x_{1} /\left(x_{1}, x_{2}\right)\right)-f\left(\left(x_{1}, x_{2}\right)\right)\right| \\
& +\left|f\left(\left(x_{1}, x_{2}\right)\right)+f\left(x_{2} /\left(x_{1}, x_{2}\right)\right)-f\left(x_{2}\right)\right| \\
\leq & \varphi\left(x_{1} /\left(x_{1}, x_{2}\right),\left(x_{1}, x_{2}\right)\right)+\varphi\left(x_{2} /\left(x_{1}, x_{2}\right),\left(x_{1}, x_{2}\right)\right) .
\end{aligned}
$$

Thus, after substituting

$$
x=x_{1} /\left(x_{1}, x_{2}\right), \quad y=x_{2} /\left(x_{1}, x_{2}\right), \quad z=\left(x_{1}, x_{2}\right),
$$

inequality (1.15) yields

$$
\begin{aligned}
& \left|f\left(x_{1}\right)-f\left(x_{2}\right)-f\left(x_{1} /\left(x_{1}, x_{2}\right)\right)+f\left(x_{2} /\left(x_{1}, x_{2}\right)\right)\right| \\
& \quad \leq \psi\left(x_{1}\right)+\psi\left(x_{2}\right)-\psi\left(x_{1} /\left(x_{1}, x_{2}\right)\right)-\psi\left(x_{2} /\left(x_{1}, x_{2}\right)\right) .
\end{aligned}
$$

We transform the expression $\sum_{i=1}^{m} \lambda_{i} f\left(x_{i}\right)$ getting rid of the terms $f\left(x_{1}\right)$, $-f\left(x_{2}\right)$ and replacing them by $f\left(x_{1} /\left(x_{1}, x_{2}\right)\right),-f\left(x_{2} /\left(x_{1}, x_{2}\right)\right)$, respectively. 
To be more precise, let us define integers $\widetilde{\lambda}_{1}, \ldots, \widetilde{\lambda}_{m+2}$ as follows:

$$
\begin{gathered}
\tilde{\lambda}_{1}=\lambda_{1}-1, \quad \tilde{\lambda}_{2}=\lambda_{2}+1, \\
\widetilde{\lambda}_{i}= \begin{cases}\lambda_{i} & \text { if } x_{i} \notin\left\{x_{1} /\left(x_{1}, x_{2}\right), x_{2} /\left(x_{1}, x_{2}\right)\right\}, \\
\lambda_{i}+1 & \text { if } x_{i}=x_{1} /\left(x_{1}, x_{2}\right), \\
\lambda_{i}-1 & \text { if } x_{i}=x_{2} /\left(x_{1}, x_{2}\right),\end{cases} \\
\widetilde{\lambda}_{m+1}=\left\{\begin{array}{ll}
0 & \text { if } \exists_{i} x_{i}=x_{1} /\left(x_{1}, x_{2}\right), \\
1 & \text { otherwise, } i=3, \ldots, m,
\end{array} \tilde{\lambda}_{m+2}= \begin{cases}0 & \text { if } \exists_{i} x_{i}=x_{2} /\left(x_{1}, x_{2}\right), \\
-1 & \text { otherwise. }\end{cases} \right.
\end{gathered}
$$

Let also

$$
x_{m+1}=x_{1} /\left(x_{1}, x_{2}\right), \quad x_{m+2}=x_{2} /\left(x_{1}, x_{2}\right) .
$$

Evidently, the $x_{i}$ 's $(i=1, \ldots, m+2)$ with $\widetilde{\lambda}_{i} \neq 0$ are pairwise different, whereas $\widetilde{\lambda}_{i}$ 's are integers satisfying

$$
\sum_{\substack{p^{k} \| x_{i} \\ 1 \leq i \leq m+2}} \tilde{\lambda}_{i}=0 \quad \text { for all } p \in \mathbb{P}, k \in \mathbb{N} .
$$

Moreover,

$$
\begin{aligned}
\sum_{i=1}^{m+2}\left|\widetilde{\lambda}_{i}\right| \omega\left(x_{i}\right)= & \left(\left|\lambda_{1}\right|-1\right) \omega\left(x_{1}\right)+\left(\left|\lambda_{2}\right|-1\right) \omega\left(x_{2}\right)+\sum_{i=3}^{m+2}\left|\widetilde{\lambda}_{i}\right| \omega\left(x_{i}\right) \\
\leq & \left(\left|\lambda_{1}\right|-1\right) \omega\left(x_{1}\right)+\left(\left|\lambda_{2}\right|-1\right) \omega\left(x_{2}\right)+\sum_{i=3}^{m+2}\left|\lambda_{i}\right| \omega\left(x_{i}\right) \\
& +\omega\left(x_{1} /\left(x_{1}, x_{2}\right)\right)+\omega\left(x_{2} /\left(x_{1}, x_{2}\right)\right) \\
= & c-2 \omega\left(\left(x_{1}, x_{2}\right)\right)<c
\end{aligned}
$$

since $p \mid\left(x_{1}, x_{2}\right)$. By our inductive hypothesis, we thus obtain

$$
\left|\sum_{i=1}^{m+2} \tilde{\lambda}_{i} f\left(x_{i}\right)\right| \leq \sum_{i=1}^{m+2}\left|\tilde{\lambda}_{i}\right| \psi\left(x_{i}\right)
$$

which does not exceed

$$
\sum_{i=1}^{m}\left|\lambda_{i}\right| \psi\left(x_{i}\right)-\psi\left(x_{1}\right)-\psi\left(x_{2}\right)+\psi\left(x_{1} /\left(x_{1}, x_{2}\right)\right)+\psi\left(x_{2} /\left(x_{1}, x_{2}\right)\right) .
$$

This estimate, jointly with (1.18) and the equality $\sum_{i=1}^{m} \lambda_{i} f\left(x_{i}\right)=\sum_{i=1}^{m+2} \widetilde{\lambda}_{i} f\left(x_{i}\right)+f\left(x_{1}\right)-f\left(x_{2}\right)-f\left(x_{1} /\left(x_{1}, x_{2}\right)\right)+f\left(x_{2} /\left(x_{1}, x_{2}\right)\right)$, yields (1.16). 
REMARK 1.9. Of course, analogous versions of Theorems 1.7 and 1.8 may be stated for complex-valued almost additive functions. The error of approximation should then be multiplied by $\sqrt{2}$.

The following is a stronger version of [7, Theorem 2] with a very simple proof.

TheOrem 1.10. Assume that a function $f: \mathbb{N} \rightarrow \mathbb{C}$ satisfies (A) and

$$
\lim _{\substack{\omega(x) \rightarrow \infty \\(r, x)=1}}(f(r x)-f(x)-f(r))=0 \quad \text { for } r \in \mathbb{S} .
$$

Then there is an arithmetic additive function $\tilde{f}$ such that $|f(x)-\widetilde{f}(x)| \leq \varepsilon$ for $x \in \mathbb{N}$. Moreover, $\left.f\right|_{\mathbb{S}}=\left.\widetilde{f}\right|_{\mathbb{S}}$.

Proof. Fix $x \in \mathbb{N}, x=r_{1} \cdot \ldots \cdot r_{s}$ with $r_{1}, \ldots, r_{s} \in \mathbb{S}$ pairwise coprime. We shall prove that $\left|f(x)-\sum_{i=1}^{s} f\left(r_{i}\right)\right| \leq \varepsilon$. For every $y \in \mathbb{N}$ we have

$$
\begin{aligned}
f(x)-\sum_{i=1}^{s} f\left(r_{i}\right)= & f(x)+f(y)-f(x y) \\
& +\sum_{i=1}^{s}\left(f\left(y r_{1} \cdot \ldots \cdot r_{i}\right)-f\left(y r_{1} \cdot \ldots \cdot r_{i-1}\right)-f\left(r_{i}\right)\right) .
\end{aligned}
$$

For any given $\eta>0$, in view of condition (1.19), we can choose $y$ coprime to $x$ and satisfying

$$
\sum_{i=1}^{s}\left|f\left(y r_{1} \cdot \ldots \cdot r_{i}\right)-f\left(y r_{1} \cdot \ldots \cdot r_{i-1}\right)-f\left(r_{i}\right)\right|<\eta .
$$

We have $|f(x)+f(y)-f(x y)| \leq \varepsilon$ and $\left|f(x)-\sum_{i=1}^{s} f\left(r_{i}\right)\right|<\varepsilon+\eta$, which ends the proof.

The technique of the proof of [7, Theorem 2] leads to the last result of this section.

TheOREM 1.11. Assume that a function $f: \mathbb{N} \rightarrow \mathbb{C}$ satisfies (A), $\left.f\right|_{\mathbb{S}}=0$ and for every sequence $\left(r_{m}\right)_{m \in \mathbb{N}}$ of mutually coprime elements from $\mathbb{S}$,

$$
\liminf _{m \rightarrow \infty} \frac{1}{m}\left|f\left(r_{1} \cdot \ldots \cdot r_{m}\right)\right|=0 .
$$

Then for every $\eta>0$ the set $\{x \in \mathbb{N}:|f(x)|<\varepsilon+\eta\}$ is large.

Proof. By Theorem 1.5 and the comments following Remark 1.6, there exists an arithmetic additive function $\widetilde{f}: \mathbb{N} \rightarrow \mathbb{C}$ such that $|f(x)-\widetilde{f}(x)| \leq$ $\sqrt{2} K \varepsilon$ for $x \in \mathbb{N}$.

Let $\left(r_{m}\right)_{m \in \mathbb{N}}$ be an arbitrary sequence of mutually coprime elements from $\mathbb{S}$. For every $m \in \mathbb{N}$ we have 


$$
\begin{aligned}
\left|\frac{1}{m} \sum_{i=1}^{m} \widetilde{f}\left(r_{i}\right)\right| & \leq \frac{1}{m}\left|f\left(r_{1} \cdot \ldots \cdot r_{m}\right)\right|+\frac{1}{m}\left|f\left(r_{1} \cdot \ldots \cdot r_{m}\right)-\widetilde{f}\left(r_{1} \cdot \ldots \cdot r_{m}\right)\right| \\
& \leq \frac{1}{m}\left|f\left(r_{1} \cdot \ldots \cdot r_{m}\right)\right|+\frac{\sqrt{2} K}{m} \varepsilon,
\end{aligned}
$$

which, in the light of our assumption, yields

$$
\liminf _{m \rightarrow \infty} \frac{1}{m}\left|\sum_{i=1}^{m} \tilde{f}\left(r_{i}\right)\right|=0 .
$$

Since (1.20) is true for every sequence $\left(r_{m}\right)_{m \in \mathbb{N}}$ of mutually coprime elements from $\mathbb{S}$, we must have

$$
\lim _{m \rightarrow \infty} \tilde{f}\left(r_{m}\right)=0 .
$$

Fix an arbitrary sequence $R=\left(x_{m}\right)_{m \in \mathbb{N}}$ of mutually coprime natural numbers such that $L:=\sup _{m \in \mathbb{N}} \omega\left(x_{m}\right)<\infty$. The conclusion of the preceding paragraph implies that

$$
\lim _{m \rightarrow \infty} \max \left\{|\tilde{f}(r)|: r \| x_{m}, r \in \mathbb{S}\right\}=0,
$$

which gives

$$
\left|\frac{1}{m} \sum_{i=1}^{m} \tilde{f}\left(x_{i}\right)\right| \leq \frac{L}{m} \sum_{i=1}^{m} \max \left\{|\tilde{f}(r)|: r \| x_{m}, r \in \mathbb{S}\right\} \stackrel{m \rightarrow \infty}{\longrightarrow} 0 .
$$

Let $R_{m}=\left\{x_{1}, \ldots, x_{m}\right\}$ for $m \in \mathbb{N}$ and denote by $R_{m}[n]$ the family of all $n$-tuples of pairwise different elements from $R_{m}$. Define

$$
M_{m}(n)=\frac{1}{\# R_{m}[n]} \sum_{A \in R_{m}[n]} f\left(\prod_{a \in A} a\right) \text { for } m, n \in \mathbb{N}, n \leq m .
$$

By the same argument as in the proof of [7, Theorem 2] we obtain

$$
\left|M_{m}(s+t)-M_{m}(s)-M_{m}(t)\right| \leq \varepsilon \quad \text { for } m, s, t \in \mathbb{N}, s+t \leq m .
$$

Since $f$ satisfies $(\mathrm{A})$ and $\left.f\right|_{\mathbb{S}}=0$, an easy induction yields

$$
\left|f\left(\prod_{a \in A} a\right)\right| \leq L n \varepsilon \quad \text { for } n \leq m, A \in R_{m}[n] .
$$

This means that the sequence $\left(M_{m}(n)\right)_{m=n}^{\infty}$ is bounded for every $n \in \mathbb{N}$ and we may consider its Banach limit $M(n):=\operatorname{LIM}_{m \rightarrow \infty} M_{m}(n)$. Inequality (1.22) yields the $\varepsilon$-additivity of the mapping $\mathbb{N} \ni n \mapsto M(n)$.

If $m, n \in \mathbb{N}$ with $n \leq m$ then

$$
\# R_{m}[n]=\frac{m !}{(m-n) !}
$$

hence

$$
M_{m}(n)=\frac{(m-n) !}{m !} \sum_{A \in R_{m}[n]}\left(\tilde{f}\left(\prod_{a \in A} a\right)+\varepsilon(A)\right)
$$


where $|\varepsilon(A)| \leq \sqrt{2} K \varepsilon$. Every $x_{i} \in R_{m}$ appears exactly

$$
\left(\begin{array}{c}
m-1 \\
n-1
\end{array}\right) n !
$$

times as a factor of $\prod_{a \in A} a$ when $A$ runs through $R_{m}[n]$. Therefore, by the additivity of $\widetilde{f}$ and formula (1.23), we have

$M_{m}(n)=\frac{(m-n) !}{m !}\left(\begin{array}{c}m-1 \\ n-1\end{array}\right) n ! \sum_{i=1}^{m} \widetilde{f}\left(x_{1}\right)+\varepsilon(m, n)=\frac{n}{m} \sum_{i=1}^{m} \widetilde{f}\left(x_{i}\right)+\varepsilon(m, n)$,

where $|\varepsilon(m, n)| \leq \sqrt{2} K \varepsilon$. By virtue of (1.21), we get

$$
M(n)=\operatorname{LIM}_{m \rightarrow \infty} \varepsilon(m, n) \in[-\sqrt{2} K \varepsilon, \sqrt{2} K \varepsilon] \quad \text { for } n \in \mathbb{N} .
$$

The function $M$, being $\varepsilon$-additive and bounded, is bounded by $\varepsilon$ (which follows, e.g., from Hyers' theorem). We may summarize the first part of the proof as follows: for an arbitrary infinite sequence of pairwise coprime natural numbers with globally bounded numbers of prime factors we have $|M(n)| \leq \varepsilon$ for all $n \in \mathbb{N}$, where $M$ is defined as above.

Now, fix $\eta>0$ and an infinite sequence $R$ with the properties described above. In the light of Lemma 1.2, it is enough to show that for at least one member $x$ of $R$ we have $|f(x)|<\varepsilon+\eta$. Suppose the contrary.

The region $\{z \in \mathbb{C}:|f(z)| \geq \varepsilon+\eta\}$ may be partitioned into finitely many parts whose convex hulls do not meet the closed ball $B(0, \varepsilon)$. There exists an infinite subsequence of $R$ whose image under $f$ lies in one of these parts. However, if $M$ is defined as above with respect to that subsequence instead of $R$, then $|M(1)|>\varepsilon$. This follows from the fact that $M(1)$ is a Banach limit of arithmetic means of complex numbers lying in a convex set disjoint from $B(0, \varepsilon)$. We have obtained a contradiction with the first part of the proof.

REMARK 1.12. Given $f: \mathbb{N} \rightarrow \mathbb{C}$ satisfying (A) we may apply the above result to $f-\widetilde{f}$, where $\tilde{f}$ is the unique arithmetic additive function such that $\left.f\right|_{\mathbb{S}}=\left.\widetilde{f}\right|_{\mathbb{S}}$. As a consequence, $f$ lies near $\widetilde{f}$ on a large subset of $\mathbb{N}$. Of course, the condition $\left.f\right|_{\mathbb{S}}=0$, after replacing $f$ by $f-\widetilde{f}$, is automatically satisfied.

REMARK 1.13. Applying the Ramsey theorem as in the proof of [7, Theorem 2] we might infer that for every $\eta>0$ the set $A_{\eta}:=\{x \in \mathbb{N}:|f(x)|<$ $\varepsilon+\eta\}$ has the following additional property: for each infinite set $B \subset \mathbb{N}$ satisfying conditions (i), (ii) of Lemma 1.2 and for each $n \in \mathbb{N}$ there exists an infinite subset $C \subset B$ such that

$$
\left\{c_{1} \cdot \ldots \cdot c_{n}: c_{i} \in C, c_{i} \neq c_{j} \text { for } i \neq j\right\} \subset A_{\eta} .
$$

However, this does not give any further information in Theorem 1.11, since every large set has this property. 
2. Stability results in the multiplicative case. Now, we are interested in functions $f: \mathbb{N} \rightarrow \mathbb{C} \backslash\{0\}$ which satisfy condition (M) with $\varepsilon \in[0,1)$. Inspired by the main ideas of the paper [2] by R. Ger and P. Semrl, we show how our results on arithmetic almost additive functions may be applied in the multiplicative case.

The following proposition is similar to [2, Corollary 2.4].

Proposition 2.1. Let $\varepsilon \in[0,1 / 4)$. Assume that a mapping $\alpha: \mathbb{N} \rightarrow \mathbb{R}$ satisfies

$$
x, y \in \mathbb{N},(x, y)=1 \Rightarrow \alpha(x y)-\alpha(x)-\alpha(y) \in \mathbb{Z}+(-\varepsilon, \varepsilon) .
$$

Then there exists a function $p: \mathbb{N} \rightarrow \mathbb{R}$ such that

$$
x, y \in \mathbb{N},(x, y)=1 \Rightarrow p(x y)-p(x)-p(y) \in \mathbb{Z}
$$

and $|\alpha(x)-p(x)| \leq K \varepsilon$ for $x \in \mathbb{N}$.

The proof is almost the same as that of [7, Proposition]. The only difference is that instead of [7, Theorem 2] we apply our Theorem 1.5. Proposition 2.1 allows us to establish the following strengthened version of $[7$, Theorem 3]. The scheme of the proof remains unchanged.

Theorem 2.2. If $f: \mathbb{N} \rightarrow \mathbb{C} \backslash\{0\}$ satisfies (M) with $\varepsilon \in[0,1)$, then there is an arithmetic multiplicative function $\tilde{f}: \mathbb{N} \rightarrow \mathbb{C} \backslash\{0\}$ such that

$$
\left|\frac{f(x)}{\widetilde{f}(x)}-1\right| \leq \delta(\varepsilon) \quad \text { and } \quad\left|\frac{\widetilde{f}(x)}{f(x)}-1\right| \leq \delta(\varepsilon) \quad \text { for } x \in \mathbb{N},
$$

where $\delta(\varepsilon)$ is a non-negative number depending only on $\varepsilon$. Moreover, $\delta(\varepsilon) \rightarrow 0$ as $\varepsilon \rightarrow 0$.

REMARK 2.3. By a careful inspection of the proof of [2, Theorem 3.4] we find that the error $\delta(\varepsilon)$ in Theorem 2.2 may be expressed by the formula

$$
\delta(\varepsilon)= \begin{cases}\sqrt{1-\frac{2 \cos (K \arcsin \varepsilon)}{(1-\varepsilon)^{K}}+\frac{1}{(1-\varepsilon)^{2 K}}} & \text { if } \varepsilon<\sin \frac{\pi}{K}, \\ 1+(1-\varepsilon)^{-K} & \text { otherwise. }\end{cases}
$$

However, if we take into account Remark 1.6, this estimate may be sharpened in the following way: for each $\eta \in(0, \pi-\arcsin \varepsilon)$ the set

$$
\left\{x \in \mathbb{N}:\left|\frac{f(x)}{\widetilde{f}(x)}-1\right| \leq \delta(\varepsilon, \eta) \text { and }\left|\frac{\widetilde{f}(x)}{f(x)}-1\right| \leq \delta(\varepsilon, \eta)\right\},
$$

where

$$
\delta(\varepsilon, \eta):=\sqrt{1-\frac{2 e^{\eta} \cos (\eta+\arcsin \varepsilon)}{1-\varepsilon}+\frac{e^{2 \eta}}{(1-\varepsilon)^{2}}},
$$


contains a subset of the form $\mathbb{N} \backslash(P \cdot \mathbb{N})$ with a finite set $P \subset \mathbb{P}$. In particular, it is a set of a positive density. Observe that $\delta(\varepsilon, \eta)$ converges to the constant appearing in [2, Theorem 3.4] as $\eta \rightarrow 0$.

The following result is dual to Theorem 1.10.

Theorem 2.4. Assume that a function $f: \mathbb{N} \rightarrow \mathbb{C} \backslash\{0\}$ satisfies (M) and

$$
\lim _{\substack{\omega(x) \rightarrow \infty \\(r, x)=1}} \frac{f(r x)}{f(x) f(r)}=1 \quad \text { for } r \in \mathbb{S} .
$$

Then there is an arithmetic multiplicative function $\tilde{f}$ such that

$$
\left|\frac{\widetilde{f}(x)}{f(x)}-1\right| \leq \varepsilon \quad \text { for } x \in \mathbb{N}
$$

Moreover, $\left.f\right|_{\mathbb{S}}=\left.\widetilde{f}\right|_{\mathbb{S}}$.

Proof. For $x \in \mathbb{N}$ with a canonical factorization $x=r_{1} \cdot \ldots \cdot r_{s}$ write

$$
\frac{\prod_{i=1}^{s} f\left(r_{i}\right)}{f(x)}=\frac{f(x y)}{f(x) f(y)} \prod_{i=1}^{s} \frac{f\left(r_{i}\right) f\left(r_{i+1} \cdot \ldots \cdot r_{s} y\right)}{f\left(r_{i} \cdot \ldots \cdot r_{s} y\right)}
$$

and argue as in the proof of Theorem 1.10.

Finally, we present a result dual to Theorem 1.7. It occurs that an appropriate "control" function, which would play the role of $(m+n) \varepsilon$ in (1.8), should be defined as $1-e^{-(m+n) \varepsilon}$. To obtain this result we repeat the scheme of the proof of [2, Theorem 3.4].

Theorem 2.5. Assume that a function $f: \mathbb{N} \rightarrow \mathbb{C} \backslash\{0\}$ satisfies

$$
\left|\frac{\prod_{i=1}^{m} f\left(x_{i}\right)}{\prod_{j=1}^{n} f\left(y_{j}\right)}-1\right| \leq 1-e^{-(m+n) \varepsilon}
$$

for every $x_{1}, \ldots, x_{m}, y_{1}, \ldots, y_{n}$ satisfying (1.9). Then there is an arithmetic multiplicative function $\widetilde{f}: \mathbb{N} \rightarrow \mathbb{C} \backslash\{0\}$ such that

$$
\frac{f(x)}{\widetilde{f}(x)} \in\left[e^{-\varepsilon}, e^{\varepsilon}\right] \quad \text { for } x \in \mathbb{N} .
$$

Proof. Write $f(x)=|f(x)| \exp (i \arg f(x))$, where $-\pi<\arg f(x) \leq \pi$. We can check that the function $x \mapsto \log |f(x)|$ satisfies the assumption of Theorem 1.7. Therefore, we get a real arithmetic additive function $g$ such that $|g(x)-\log | f(x)|| \leq \varepsilon$ for $x \in \mathbb{N}$.

By our supposition, we have

$$
x, y \in \mathbb{N},(x, y)=1 \Rightarrow\left|\left(\frac{f(x y)}{f(x) f(y)}\right)^{n}-1\right| \leq 1 \quad \text { for } n \in \mathbb{N},
$$


thus

$$
x, y \in \mathbb{N},(x, y)=1 \Rightarrow \frac{f(x y)}{f(x) f(y)} \in \mathbb{R} .
$$

In other words, the function $x \mapsto \arg f(x)$ is arithmetic additive modulo the set $2 \pi \mathbb{Z}$. A moment's reflection shows that it has to be the sum of an arithmetic additive mapping and a $2 \pi \mathbb{Z}$-valued mapping.

Define $\widetilde{f}(x)=\exp (g(x)+i \arg f(x))$ for $x \in \mathbb{N}$. Then $\tilde{f}$ is arithmetic multiplicative and

$$
\frac{f(x)}{\widetilde{f}(x)}=\frac{\exp g(x)}{|f(x)|} \in\left[e^{-\varepsilon}, e^{\varepsilon}\right] .
$$

\section{References}

[1] R. Ger, Superstability is not natural, Rocznik Naukowo-Dydaktyczny WSP w Krakowie Prace Mat. 159 (1993), 109-123.

[2] R. Ger and P. Šemrl, The stability of the exponential equation, Proc. Amer. Math. Soc. 124 (1996), 779-787.

[3] G. H. Hardy and S. Ramanujan, The normal number of prime factors of a number n, Quart. J. Math. 48 (1917), 76-92.

[4] D. H. Hyers, On the stability of the linear functional equation, Proc. Nat. Acad. Sci. USA 27 (1941), 222-224.

[5] N. J. Kalton and J. W. Roberts, Uniformly exhaustive submeasures and nearly additive set functions, Trans. Amer. Math. Soc. 278 (1983), 803-816.

[6] J. L. Kelley, Measures on Boolean algebras, Pacific J. Math. 9 (1959), 1165-1177.

[7] T. Kochanek, Stability aspects of arithmetic functions, Acta Arith. 132 (2008), $87-98$.

[8] T. Kochanek and M. Lewicki, Stability problem for number-theoretically multiplicative functions, Proc. Amer. Math. Soc. 135 (2007), 2591-2597.

[9] N. Pippenger, Superconcentrators, SIAM J. Comput. 6 (1977), 298-304.

[10] R. T. Rockafellar, Convex Analysis, Princeton Univ. Press, Princeton, NJ, 1970.

[11] S. M. Ulam, Problems in Modern Mathematics, Wiley, New York, 1960.

Institute of Mathematics

Silesian University

Bankowa 14

40-007 Katowice, Poland

E-mail: tkochanek@math.us.edu.pl

Received on 19.11.2008

and in revised form on 28.1.2009 